\title{
Estimating Low-power Radio Signal Attenuation in Forests: A LiDAR-based Approach
}

\author{
Silvia Demetri, Gian Pietro Picco, and Lorenzo Bruzzone \\ Department of Information Engineering and Computer Science (DISI), University of Trento, Italy \\ \{silvia.demetri, gianpietro.picco, lorenzo.bruzzone\}@unitn.it
}

\begin{abstract}
Wireless sensor networks offer unprecedented opportunities to monitor natural ecosystems. However, despite the growing number of applications (e.g., forest fire detection, wildlife monitoring), the deployment challenges posed by the real-world natural environment still hinder the widespread adoption of this technology. In particular, the unpredictability of the low-power wireless channel in the presence of vegetation requires costly trial-and-error pilot campaigns to understand where and how to place the wireless nodes. In this paper, we propose a technique based on remote sensing for accurately estimating low-power radio signal attenuation in forest environments. We leverage airborne Light Detection and Ranging (LiDAR) instruments and related automatic data analysis systems to determine local forest attributes (e.g., tree density) that, once factored into a specialized radio path loss model, enable accurate estimation of the received signal power. Our approach is i) automatic, i.e., it does not require in-field campaigns, and $i i)$ fine-grained, i.e., it enables perlink estimates. Our validation from deployments in a real forest shows that the error of our per-link estimates of the received signal power is around $\pm 6 \mathbf{d B m}$ - the accuracy of RSSI readings from the radio transceiver.
\end{abstract}

Keywords-Low-power wireless communication, wireless sensor networks, remote sensing, LiDAR, IEEE 802.15.4.

\section{INTRODUCTION}

The last decade witnessed a surge of applications where low-power wireless communication is employed to monitor natural ecosystems. In most cases, wireless sensor networks (WSNs) are deployed to either study a given environment [1] or habitat [2], or to protect it by detecting hazards such as forest fires [3]. Recently, however, low-power wireless has been employed also as a means to detect proximity among the wildlife that dwells in the natural environment [4], [5].

To these scenarios, low-power wireless communication brings unprecedented flexibility. However, it also brings a great deal of complication, due to the fact that its performance, critical to system operation, are strictly dependent on the specific environment. For instance, in the former case of a WSN with fixed nodes, its planning (number and position of nodes) must take into account the target environment and its effect on network performance. However, a similar issue arises also in the aforementioned proximity detection studies where the animal-borne low-power wireless nodes act as a "proximity sensor", recording beacons exchanged among nodes. Again, the distance at which detection may occur strongly depends on the environment, and changes based on the movement patterns of the animal; knowledge about its impact is key to enable a correct scientific inference from proximity data.

These problems are exacerbated in the forest environment, our focus in this paper, where the heterogeneous vegetation creates local effects that amplify complexity. This longstanding issue is a barrier to environmental scientists willing to adopt WSN technology, leaving them with the sole option of engaging in effort-demanding pilot deployments [6]-[8].

Indeed, despite the large literature on radio propagation in the presence of vegetation, the problem of deriving accurate estimates for low-power wireless is still largely unsolved. The majority of existing approaches rely on distance as the main parameter affecting performance, implicitly assuming a homogeneous environment in the target area. However, it is well-known that this assumption does not hold in general for low-power wireless [9]. In the case of forests, vegetation and its heterogeneity (in species and/or density) are the dominant factor determining performance, as confirmed empirically by a few studies. Marfievici et al. [7] report significant differences among species, assessed by directly immersing the same WSN deployment (i.e., same nodes and topology) in different forests. Liu et al. [8] observe, in a single large-scale deployment, that despite the regular placement of nodes, the irregularity of the forest makes the radio signal propagation irregular, and the network behavior largely unpredictable.

These aspects are only partly accounted for in existing radio propagation models tailored to forests, which carry two main limitations:

1) they assume a priori knowledge of vegetation attributes, whose estimation process remains often undefined, and in practice is commonly performed by means of costly in-field observations - therefore, the problem is simply moved to a different layer;

2) the estimation of vegetation attributes is typically coarsegrained, spanning relatively large areas; in principle, instead, a fine-grained estimate on a per-link basis would be desirable, given the high level of variation observed in the forest environment.

In this paper, we propose a solution to both problems based on remote sensing, a technology already applied to forest monitoring. Remote sensing systems, typically satellitebased or airborne, acquire data (e.g., images) over wide areas and, through signal processing techniques, enable fine-grained, automatic estimation of tree and forest attributes. Here, we focus on a specific technology, Light Detection and Ranging (LiDAR), whose characteristics we concisely summarize in Section II. The use of LiDAR data allows us to:

1) estimate forest attributes through automatic data analysis, therefore removing the need for in-field campaigns;

2) derive estimates that are very precise, to the point of 
identifying, e.g., the position of each individual tree and its diameter. We exploit this rich information to enable fine-grained estimates on a per-link basis, i.e., accounting for the presence of trees on each link.

Our approach is described in Section III. The starting point is an existing path loss model [10], itself an extension of the common log-normal one, that i) takes explicitly into account the impact of trees on communication, and ii) assumes that the latter occurs at trunk level-a common choice in several of the aforementioned WSN deployment scenarios. The model in [10] relies on a vegetation index (VD) that depends on the average tree density and diameter throughout the area of interest, that are assumed to be known a priori. Our first contribution is the definition of a processing chain that, based on LiDAR data, automatically and accurately estimates these forest attributes. However, we show that, based on these attributes, significantly better estimates of the received signal power can be achieved. Instead of averaging these attributes over a macro-area, we perform a fine-grained analysis where the impact of trees is ascertained only around each individual link, therefore enabling more accurate, per-link estimates. In particular, this allows us to determine whether a link enjoys a clean line of sight, and therefore a free space path loss model is a better fit, or instead it is obstructed by trees.

The accuracy of our approach in general, and of per-link estimates in particular, is evaluated in Section IV based on smallscale WSN deployments where we gather RSSI (Received Signal Strength Indicator) traces in an area where both LiDAR data and human-derived ground truth are available. Our results show that the accuracy we obtain with our per-link approach is significantly superior to existing approaches, including the model in [10], automatically tuned with our LiDAR-based approach. Specifically, accuracy is largely within $\pm 6 \mathrm{dBm}-$ the accuracy of RSSI readings from the radio transceiver.

The paper is completed by a concise survey of the state of the art in Section V, followed in Section VI by a discussion of opportunities for future work on the topic of this paper.

\section{Background: Remote Sensing Systems}

Remote Sensing (RS) systems acquire data and images over wide areas of the globe by means of sensors, usually mounted on satellites or airplanes, which measure the electro-magnetic radiation reflected by the surface under investigation. From this indirect measure, different properties of the reflecting objects can be derived, depending on the type of system and its specific characteristics [11]. By processing RS data a wealth of information about the target surface can be conveniently retrieved since i) signal and image processing techniques enable automatic and scalable analysis, and $i$ ) the cost of data and information extraction procedures is limited w.r.t. ground campaigns. RS is exploited for several applications including forest analysis, for which the systems commonly used are Light Detection and Ranging (LiDAR) and optical instruments.

LiDAR is an active system where the source of radiation (i.e., laser pulses) is generated by the system itself. A laser scanner, usually mounted on an airplane, transmits pulses with a nadir-looking geometry (i.e., towards the scene under investigation). Each pulse hits objects (e.g., trees) at different heights during its propagation, generating a reflection (called return) at every hit, that backpropagates towards the scanner. The time elapsed between the transmission of the pulse and the reception of its reflected component is converted into a distance estimate. By exploiting this mechanism, shown in Figure 1, LiDAR represents objects in a three-dimensional space. The representation can be very precise in both the horizontal and vertical directions, allowing one to retrieve information about shape, size, and position of trees. Indeed, LiDAR data has been extensively used for the estimation of forest structural parameters both at stand level (i.e., by considering groups of trees) [12], [13] and single-tree level [14], [15] (e.g., trunk diameter estimation). The precision of the representation is strictly dependent on the spatial density of the emitted laser pulses. The most accurate estimates of forest parameters are achieved with high-density LiDAR data (i.e., $>5$ points $/ \mathrm{m}^{2}$ ).

In contrast, optical instruments represent only the horizontal structure of forests, providing little information about the vertical one. Moreover, being passive systems operating in the optical frequency bands, they are strongly affected by acquisition conditions (i.e., lighting, atmosphere, topography and geometry) affecting the reliability of information extraction. Optical images (i.e., multispectral and hyperspectral images) can be an alternative to LiDAR data, since their lower precision is compensated by a lower cost. Further, the two technologies can be seen as complementary. The spectral information provided by optical systems is related to the material and nature of the objects themselves and, depending on the spatial and spectral resolution, may allow us to identify forest areas, classify tree species, or quantify the amount of green leaves in the canopy [16], [17]. The work in [18] explores the joint use of the two technologies, studying the tradeoffs between cost of data and accuracy of the forest parameter estimation.

In this work we consider only LiDAR due to the high level of detail it provides w.r.t. forest structural description (both horizontal and vertical); the possibilities opened by its integration with optical data is part of our planned future work.

\section{APPROACH}

Several models for radio propagation through vegetation have been proposed, both mechanistic [19] and empirical [10], [20]-[23], as discussed in Section V. The work we describe here is based on the empirical model in [10], as it shares our focus on communication at the trunk level. This model is an extension of the log-normal path loss model, where its parameters are expressed as a function of local forest attributes, based on a large set of measurements in forests with different species.

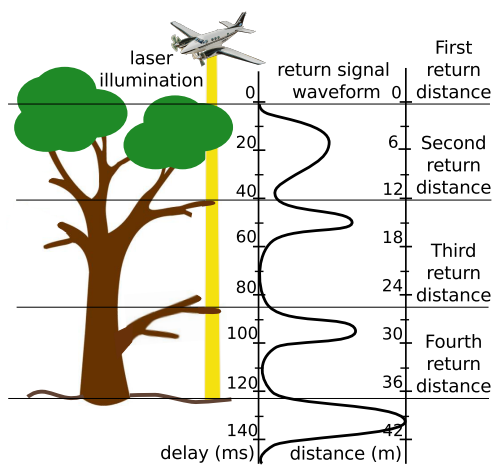

Fig. 1. Airborne LiDAR acquisition mechanism. 
However, this model (and most of the literature) assumes that vegetation is uniform in the area where radio attenuation must be estimated-an overly optimistic assumption that leads to imprecise estimates of the connectivity among individual links.

In the rest of this section we illustrate our remote sensing approach, based on LiDAR data and signal processing techniques. We overcome the two limitations above by:

1) estimating the model coefficients via automatic forest attribute extraction, which can be reliably performed over large areas without in-field campaigns;

2) providing fine-grained, per-link analysis of radio signal attenuation, greatly improving the overall accuracy of the resulting estimates, as shown quantitatively in Section IV.

\section{A. Radio Signal Propagation in a Forest}

The widely adopted log-normal path loss model [24]

$$
P L[d B]=P L\left(d_{0}\right)+10 \cdot n \cdot \log \left(\frac{d}{d_{0}}\right)+X_{\sigma}
$$

describes both the logarithmic decay of the average signal power as a function of the distance $d$ from a transmitter and the random variation of the received power around the average. The level of attenuation in the signal power is modeled as a function of three main parameters: i) $P L\left(d_{0}\right)$, the path loss at a known reference distance $d_{0}$ in the far field; $i$ i) $n$, the path loss exponent representing the attenuation rate w.r.t. distance; iii) $\sigma$, the standard deviation of a zero-mean Gaussian random variable $X$ representing the variation around the average.

These parameters are strictly dependent on the environment at hand. Their value is difficult to determine in general due to the wide variability of the characteristics of the environment itself. In our case, trees are known to cause scattering, reflection, diffraction and absorption phenomena, depending on the radio frequency, the size of trunks, branches and leaves, and the path interception materials. Therefore, these parameters are usually estimated empirically, by curve fitting on large amounts of measurements collected in the target location [23].

Azevedo et al. [10] observed a linear relation between the path loss model parameters and the local vegetation characteristics. For instance, for our frequency of interest $f=2.4 \mathrm{GHz}$, the parameters of the log-normal path loss model take the form:

$$
\begin{aligned}
P L\left(d_{0}\right) & =-0.82 \cdot V D+40.1 \\
n & =0.1717 \cdot V D+2.2043 \\
\sigma & =4.4
\end{aligned}
$$

where path loss parameters are expressed as a linear function of a vegetation index $V D$ defined exclusively as a function of detailed local vegetation attributes. Indeed, $V D$ is defined as

$$
V D=T D \cdot D
$$

where $T D$ [trees $/ \mathrm{m}^{2}$ ] is the average density of trees, and $D[\mathrm{~cm}]$ is the average diameter of their trunks.

This is a significant advancement w.r.t. the problem of predicting the impact of vegetation on wireless communication. However, one key piece of information enabling the practical application of this model is missing in [10]: how to estimate the vegetation index $V D$ reliably and cheaply. This is precisely what we address next, as one of the contributions of our work.

\section{B. Determining Forest Attributes with LiDAR}

We estimate the average diameter $D$ and density $T D$ of trees in an automatic fashion by processing high-density raw LiDAR data. The latter are actually LiDAR returns, which can be visualized as three-dimensional point clouds. An example is shown in Figure 3a, where each point represents the height at which the forest trees or ground were hit by the laser pulse.

These raw LiDAR data points are processed automatically, yielding a map in which each individual tree is represented, along with its attributes of position and trunk diameter. The processing, shown in Figure 2, unfolds through three main phases, described next:

1) pre-processing: it extracts a digital Canopy Height Model (CHM), i.e., a high resolution raster dataset that maps the tree height as a discrete surface;

2) single-tree identification: the crown of each tree is determined by properly segmenting the CHM raster image;

3) estimation of forest attributes: the position and dendrometric attributes (i.e., height, crown radius, and trunk diameter) of each tree are determined.

Preprocessing. The LiDAR raw data are pre-processed in two steps. First, the Digital Elevation Model (DEM) is subtracted from each raw data point. The DEM represents the terrain and its morphology, mapping each pixel to the height of the terrain, with a precision that depends on the resolution of the technique used. The DEM is derived by the LiDAR data according to a standard technique [25]. This operation allows us to adjust and correct the raw data by extracting the actual elevation from the ground of each point. Second, from these corrected data points we generate a raster image with a geometrical resolution of $50 \mathrm{~cm}$ (i.e., each pixel represent a $50 \times 50 \mathrm{~cm}^{2}$ ground area) by assigning to each pixel the maximum height value of the points (as obtained from the previous step) belonging to the corresponding area. The output is the CHM raster image.

Single-tree identification. The next step is to detect each tree in the site under investigation, and to delineate its crown. To this end we apply a segmentation technique similar to [12]. We first perform a convolutional prefiltering on the CHM, to emphasize local maxima and tree crowns. Then, we apply the set level method [15] to detect local peaks and identify the tree tops. The latter represent the seeds we use to initialize a segmentation procedure based on the region growing technique. In each step, the region around each seed is expanded by including all the neighboring pixels. The process is iterated as long as the canopy height value of those pixels is higher than a predefined threshold (i.e., the height is decreased less than $80 \%$ w.r.t. the seed) and the region diameter does not exceed a maximum acceptable value, $15 \mathrm{~m}$ in our case.

The output of this processing stage is a tree map representing the dominant layer of the forest with a $50 \mathrm{~cm}$ spatial

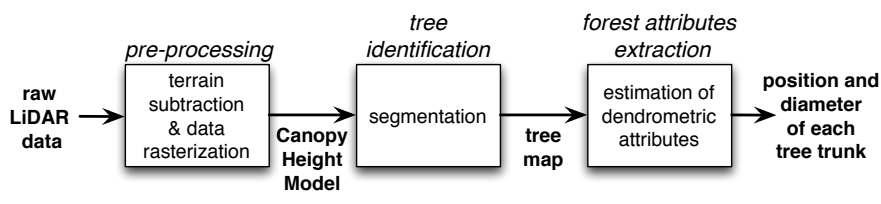

Fig. 2. Extracting tree attributes from raw LiDAR data. 


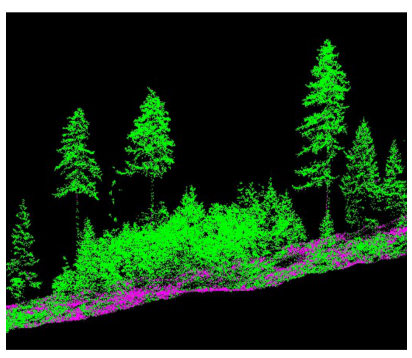

(a)

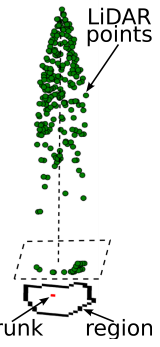

(b)

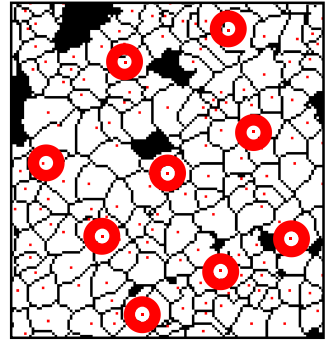

(c)
Fig. 3. (a) High-density LiDAR data sample (b) LiDAR points corresponding to a single tree (c) Tree map example

resolution. Each tree crown in our scene is delineated and the position of the trunk, corresponding to the position of the tree top, is estimated. An example showing the relation between the source raw LiDAR data and the resulting region delineating the crown is shown at the bottom of Figure $3 \mathrm{~b}$; the position of the tree trunk is the dot inside the region. Figure $3 \mathrm{c}$ shows an example tree map containing several of these regions (i.e., crowns and trunks). The area shown is actually one we used in the experiments we describe in Section IV; the bold circles represent the position of WSN nodes.

Estimation of forest structural attributes. The tree map allows us to determine the average density of trees $T D$, as number of trees per square meter. Moreover, it provides information about the position of those trees, which is exploited next to derive our per-link estimates of the radio signal power.

However, a last processing step is necessary to extract the diameter $D_{t}$ of each tree $t$. We estimate the Diameter at Breast Height (DBH) of each tree trunk using the approach presented in [14]. We model the diameter as a function of the tree height $H_{L}$ and crown radius $K_{L}$ as follows:

$$
D_{t}=b_{0}+b_{1} H_{L}+b_{2} K_{L}+b_{3} H_{L}^{2}+b_{4} K_{L}^{2}
$$

The tree height $H_{L}$ can be easily derived directly from the CHM value corresponding to the tree top pixel (or seed). Indeed, in the previous step we directly map this value to the maximum height of the pre-processed LiDAR points belonging to the corresponding $50 \times 50 \mathrm{~cm}^{2}$ area. The crown's horizontal area is approximated by the area covered by all the pixels belonging to the corresponding region, from which the crown radius $K_{L}$ is easily computed as the radius of the circle whose area is equivalent to the region area.

We determine the coefficients $\left\{b_{0}, \ldots, b_{4}\right\}$ by applying a multi-linear regression that relates a small set of ground truth measurements of tree heights, collected by the local forest service in the location where we performed our experiments, with the tree height and crown radius, namely, with $H_{L}, K_{L}$, $H_{L}^{2}$, and $K_{L}^{2}$. We can then apply these coefficients to Eq. (4) and estimate the diameter of all the trees belonging to the entire target forest site.

It is worth noting that in-field tree measurements are not strictly necessary, as other diameter estimation approaches exist that do not rely on them. One example are the heightdiameter allometric equations widely adopted for forest inventories, which relate the tree dimensions to each other according to the specific tree species.

\section{Automatic Model Tuning and Link-Level Estimate}

We now describe how we exploit the automatic processing just described towards building estimates of the radio signal power in the target forest site. We distinguish two cases, we hereafter refer to as AREA and LINK. AREA refers to estimates derived using the original model by Azevedo et al. [10], which considers a single vegetation index $V D$ across the area at hand (i.e., the entire area being considered for the deployment). The contribution we put forth here is the automatic computation of $V D$. LINK, instead, refers to our own adaptation of this model, enabling more accurate per-link estimates, for which the vegetation index $V D$ is individually computed.

AREA model. We exploit the output of the processing of LiDAR data to automatically compute the index $V D$, necessary to derive estimates based on the model in [10].

We compute the tree density $T D$ by simply counting the overall number of trunks determined in the previous step and dividing by the area of interest. Similarly, we easily compute the average diameter $D$ based on the individual diameter estimates $D_{t}$. The vegetation index $V D$ is simply the product of $T D$ and $D$, as per Eq. (3); substituting the value of $V D$ in Eq. (2) yields the value of path loss coefficients; substituting the latter in Eq. (1) yields the expected path loss $P L$ in the target area, i.e., the expected amount of attenuation in the signal power depending on the distance.

The interesting quantity from an engineering point of view, however, is the expected received power $P^{r x}[\mathrm{~dB}]$. This can be computed as a function of path loss, at given distance and for a given frequency, by the following

$$
P^{r x}=P^{t x}+G^{t x}+G^{r x}-P L
$$

where $P^{t x}$ is the transmission power, and $G^{t x}$ and $G^{r x}$ the receiving and transmitting antenna gains, respectively.

In other words, Eq. (5) enables us to predict, in a given forest area, the received power as a function of distance.

LINK model. The fact that we are able to obtain automatically predictions of received power using AREA is already per se a significant advancement of the state of the art. Nevertheless, the AREA model is rather coarse, as it assumes that the influence of vegetation is homogeneous across the target area. Unfortunately, it is well-known that typical forest vegetation can not be treated as a single homogeneous dielectric mean [22] and that vegetation irregularity causes, in turn, signal propagation to be irregular [8], leading to non-uniform link connectivity even with a uniform placement of nodes.

Interestingly, AREA does not exploit all the information that can be extracted from the processing of raw LiDAR data. Only the average tree density and average tree diameter are considered, as prescribed by Eq. (3), while our processing actually yields the individual position of each tree $t$, along with its diameter $D_{t}$. Next, we show how this information can be exploited into a link-level model which takes into account the forest attributes on a per-link basis, i.e., between each node pair, leading to the significant accuracy improvements we discuss in Section IV.

The idea behind our LINK model is very simple, yet very effective. It exploits the detailed knowledge about the position 


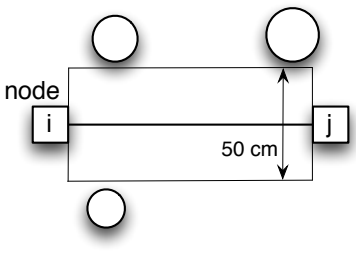

(a) Clean-LOS

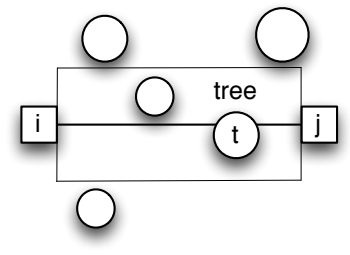

(b) Obstructed-LOS.
Fig. 4. Determining the presence of trees on the line of sight.

and diameter of each tree to compute a vegetation index $V D_{i j}$ specific to the link between node $i$ and $j$ :

1) if $V D_{i j}=0$, the link enjoys a clean line of sight: therefore, the link behavior is better approximated by the free space path loss model

$$
P L_{\text {free }}[d B]=20 \log (d)+20 \log (f)-27.55
$$

where $d[\mathrm{~m}]$ is the distance and $f[\mathrm{MHz}]$ the frequency.

2) otherwise, if $V D_{i j} \neq 0$, the behavior of the link is better estimated by a "localized" version of Eq. (5) where the area-specific $V D$ is replaced by the link-specific $V D_{i j}$.

Figure 4 illustrates the concept. $V D_{i j}=T D_{i j} \cdot D_{i j}$ is computed only within the rectangular area (hereafter called the link area) whose length is the line connecting the nodes $i$ and $j$ composing the link, and whose height is the pixel resolution used for processing, i.e., $50 \mathrm{~cm}$ in this paper. $T D_{i j}$ is trivially computed by dividing the number of trees in the link area by the area of the latter, while $D_{i j}$ is simply the average diameter computed over the trees in the link area. If there are no trees in the link area $\left(T D_{i j}=0\right)$, the link enjoys a clean line of sight (clean-LOS) as shown in Figure 4a. Otherwise, if $V D_{i j} \neq 0$, some trees are contained in the aforementioned area, and the link is obstructed (obstructed-LOS) as shown in Figure $4 \mathrm{~b}$. Since the notion of obstruction is defined by considering the entire link area, a link may be obstructed even if no tree is actually sitting on the line connecting the two nodes, as in the case where tree $t$ did not exist in Figure $4 \mathrm{~b}$. The actual processing to classify links based on line of sight is very simple, and simply consists of checking whether the set of pixels belonging to the link area intersects the set of pixels in which the positions of trunks are mapped.

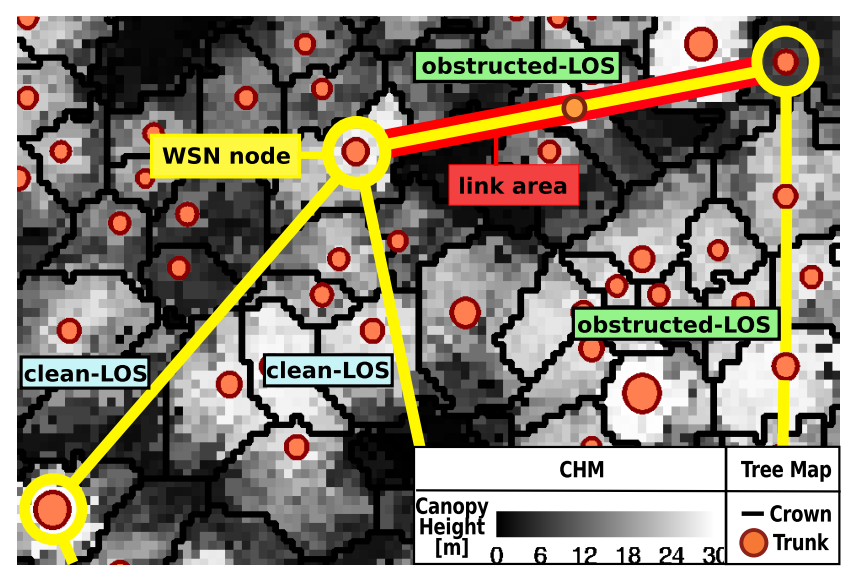

Fig. 5. Classifying links based on line of sight: A real example.
Figure 5 shows a detail of a real tree map representing the forest site where we performed the experiments reported in Section IV. A portion of the WSN we deployed in that location is also depicted: the bold circles denote the positions of nodes (attached to trunks), while thick lines denote some of the links connecting them.

\section{VALIDATION}

We validate our LiDAR-based approach for the automatic assessment of low-power radio signal attenuation in forests by comparing our predictions with RSSI traces we collected in small-scale WSN deployments in a real forest. First, we present the selected location and describe our WSN deployments, the LiDAR data set we used, and how we acquired in-field RSSI traces. Then, we report and discuss our experimental results.

\section{A. Experimental Location}

Our study area is a typical alpine forest site in Val di Sella (Trentino, Italy), $1000 \mathrm{~m}$ above sea level. It is a mixed forest with trees belonging to the European beech (Fagus sylvatica) and Norway spruce (Picea Abies) species. No understory is present, the green-leaved part is composed by only a single layer of canopy. We selected this location because it is of particular interest for the local forest service. Therefore, this choice allowed us to exploit the availability of both highdensity LiDAR data and, for validation, ground truth data collected in-field by the forest service itself.

We consider two different land plots in our study, shown in Figure 6a, each with an extension of $\sim 1700 \mathrm{~m}^{2}$. One is located in the middle of the forest, and the geographical position of its central point is $46^{\circ} 0^{\prime} 53.64^{\prime \prime} \mathrm{N}, 11^{\circ} 22^{\prime} 2.51^{\prime \prime} \mathrm{E}$. We refer to this as internal plot. The second site, which we refer to as the edge plot, is on the edge of the forest, close to a clearing, with center at $46^{\circ} 0^{\prime} 47.45^{\prime \prime} \mathrm{N}, 11^{\circ} 25^{\prime} 52.16^{\prime \prime} \mathrm{E}$.

\section{B. LiDAR Dataset and Related Processing}

The LiDAR dataset we use has been acquired by an Optech ALTM 3100EA sensor mounted on an airborne platform. The laser scanner emits pulses with wavelength $1064 \mathrm{~nm}$. For each pulse, up to four returns (i.e., points associated up to the fourth object hit by the laser pulse) were recorded, providing an average point density $>5 \mathrm{pt} / \mathrm{m}^{2}$. This dataset has been acquired

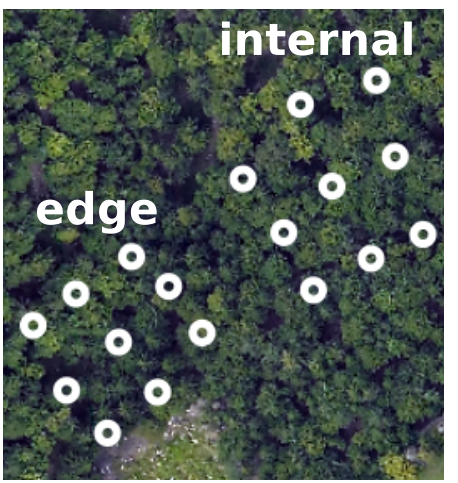

(a)

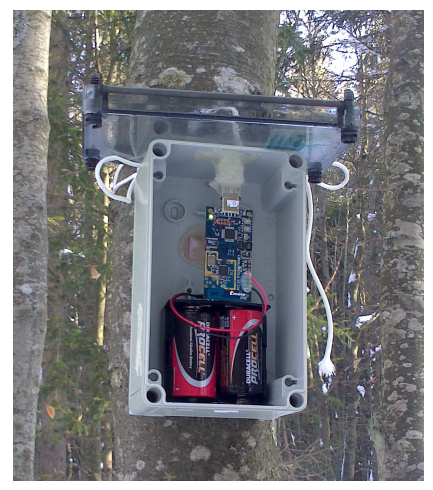

(b)
Fig. 6. (a) Experimental location and network topologies. (b) Node setup. 
over our target location on September 4, 2007. Given that our target is a dense old-growth forest, it is reasonable to assume that its structure at trunk level is not changed significantly between the LiDAR data acquisition and our RSSI traces collection. This assumption has been confirmed also by the local forest service.

By applying to the raw data the processing chain described in Section III-B we obtain a tree map for the whole area comprising both the internal and edge plots. We visually evaluate the accuracy of the obtained map by overlapping it both with the CHM and with an ortophoto, shown in Figure 6a, representing the same area with the same resolution. We assessed a good agreement between the crowns delineated in our tree map and the trees discernible in the other images.

We quantitatively evaluate the precision of the tree map and of the extracted tree attributes by exploiting the availability of a set of measurements gathered in our internal study site by the local forest service, which performed forest inventory by surveying trees in sample locations. One of these surveys targets a circular land plot with a $20 \mathrm{~m}$ radius, enclosed in our internal site. The forest service mapped 35 trees in this plot, noting their species along with height and trunk diameter at breast height. In comparison, in the same circular plot our processing chain detected 37 trees, of which 32 correspond to an actual one (i.e., $91 \%$ of trees are correctly detected). More precisely, we obtained 5 wrong detections or false positives (i.e., tree tops which actually do not correspond to trunks) and 3 missed detections or false negatives (i.e., missed tree tops/trunks). For the same set of trees, the multilinear regression we applied for the estimation of trunk diameters gives a coefficient of determination $R^{2}=0.7$ and a Root Mean Squared Error $R M S E=2.75 \mathrm{~cm}$ w.r.t. ground truth values.

As a consequence, the difference between the estimated and ground truth values of $T D, D$ and $V D$ is 0.0016 trees $/ \mathrm{m}^{2}$, $0.45 \mathrm{~cm}$, and 0.02 , respectively. This translates into a difference in the estimated received signal power $<0.05 \mathrm{dBm}$ for link distances in the range $[0,60] \mathrm{m}$. This difference can be safely considered negligible in our context, as it is well below the RSSI reading accuracy of the radio chip. Therefore, we conclude that our raw LiDAR data allows us to estimate the relevant vegetation parameters with adequate precision.

\section{Low-power Wireless RSSI Traces and Their Collection}

The RSSI traces were collected during November 2013, with sunny weather. We deployed the same WSN in both forest plots and collected traces from 12:30 to 14:30 in the internal plot, and from 15:00 to 17:00 in the edge plot. During the experimental campaign we placed a LASCAR EL-USB-2+ data logger in the same site where we deployed the network to sample both temperature and relative humidity every 15 minutes, to accurately monitor the meteorological conditions experienced by the network. Temperature and humidity were almost stable during each time interval, ranging from $-2.5^{\circ} \mathrm{C}$ to $-3^{\circ} \mathrm{C}$, and from $35 \%$ to $30 \%$, in the internal plot, and from $-3^{\circ} \mathrm{C}$ to $-4^{\circ} \mathrm{C}$ and from $30 \%$ to $25 \%$ in the edge plot, respectively.

Node placement. The WSN we deployed is composed of 9 nodes, placed in a $3 \times 3$ grid topology, to characterize the connectivity in a square portion-a sample "forest slice"of our target environment. Nodes were powered by D-size batteries and placed in waterproof boxes. Care was taken to ensure the same vertical mounting (i.e., antenna orientation) for all nodes while placing them in boxes. Boxes were then latched onto tree trunks with elastic bands at $1.7 \mathrm{~m}$ from the ground, as depicted in Figure 6b. Since boxes were attached to trees, and these hardly ever form a perfect grid, the actual topology (Figure 6a) only approximated the intended one.

Hardware platform. We use the popular TMote Sky hardware platform, operating within the ISM $2.4 \mathrm{GHz}$ frequency band according to the IEEE 802.15.4 standards. The radio module mounted on the platform is ChipCon 2420, which includes a digital Direct Sequence Spread Spectrum (DSSS) baseband modem coupled with a digital offset-QPSK modulator, providing an effective data rate of $250 \mathrm{kbps}$. We exploit the integrated inverted-F microstrip antenna, which is pseudoomnidirectional with gain of $3.1 \mathrm{dBi}$.

Software platform. The in-field collection of RSSI traces was performed using TRIDENT [26], a tool developed in our group for the untethered execution of communication experiments and collection of connectivity traces. The tool automatically produces the TinyOS code to be installed on TMote Sky motes, based on the experiment configuration input by the user.

The communication links are probed by exchanging radio messages. More precisely, each mote broadcasts a message in a round-robin fashion, to avoid collisions, while all the others are listening to the radio channel. Each time a message is received the reception event is locally recorded by the receiving node, together with the corresponding RSSI value. In addition, nodes sample and locally store the ambient noise floor level. The same procedure is repeated until a user-configured number of messages is sent by each network node. The completion of this process defines a round. Each round is characterized by a set of parameters - time interval between two consecutive transmissions or Inter Packet Interval (IPI), transmission power, radio channel-that can be set and configured in the experiment design phase. The results of the experiment (i.e., the sequence of packet receptions and their quality indicators) are stored in the local memory and can be recovered by means of both multi-hop forwarding among network nodes or direct USB connection.

Experiment setup and execution. Our experiment is composed of four 30-minutes rounds, in which each node of the network sends 200 messages with IPI equal to 9 s. All nodes play both sender and receiver roles. The result is a roundrobin sending process in which at every second one node is transmitting a packet, while the others are listening. We choose to keep an interval of $1 \mathrm{~s}$ between transmissions to avoid possible clock drifts that can cause collisions among senders, given that we are using no Medium Access Control (MAC) protocol. Nodes always transmit on channel 18 (i.e., at 2.44 $\mathrm{GHz}$ with a bandwidth of $3 \mathrm{MHz}$ ). We alternate rounds with transmission power $-1 \mathrm{dBm}$ and $-8 \mathrm{dBm}$, which we refer to as high power and low power respectively.

This experimental setting allows us to probe each $l i n k_{i j}$ (i.e., the link from node $_{i}$ to node $_{j}$ ) every $9 \mathrm{~s}$; we collect the raw packets, along with per-round and overall statistics. We expected to probe 72 links for each site, considering separately 
$\operatorname{link}_{i j}$ and $l i n k_{j i}$. However, the actual number of links we probed (i.e., 30 for each site) is lower than expected because some nodes malfunctioned halfway through the experiments, and were therefore excluded from the analysis. For each $l i n k_{i j}$ the traces we collect consist of a sequence of records for each round. Each record represents the reception of a message along that link and contains RSSI and noise floor related to the reception of that message. The results in this paper are based on the analysis of the 41,794 data points we collected overall.

\section{Results}

In this section, we present our experimental results, validating our approach against the actual received signal power measured in our WSN deployment. Our goal is to assess to which extent: $i$ ) conventional empirical channel models for vegetated environments match the real RSSI traces we collected at trunk level; ii) LiDAR data allows us to automatically describe and represent local vegetation characteristics and enable received signal power predictions in a forest area; iii) we can improve the prediction accuracy by analyzing trees configuration and its impact at per link level.

The RSSI values in our traces represent the sum of the received radio signal power and the noise power. Therefore, we can convert those values to a received power indicatorcomparable with the predictions of the models-and compute the corresponding $P^{r x}$ by subtracting, in Watt scale, the noise floor level from RSSI. This step, in addition, makes our analysis independent from possible hardware differences, in terms of noise figure, between nodes.

Shortcomings of conventional empirical channel models. As a term of comparison, we first estimate the path loss $P L$ by applying both the Weissberger model [21] and the COST 235 model [20]. These conventional models estimate the excess attenuation due to vegetation as a quantity $L_{v e g}[\mathrm{~dB}]$. The overall path loss in this model is obtained as $P L_{\text {free }}+L_{\text {veg }}$, where the first term is the free space path loss as per Eq. (6). The excess attenuation $L_{v e g}$ is expressed as a function of the radio frequency $f$, in $\mathrm{GHz}$, and the depth of foliage $d$, in meters. For Weissberger, the excess attenuation is

$$
L_{\text {veg } W}[d B]= \begin{cases}0.45 f^{0.284} d & 0 \leq d \leq 14 \\ 1.33 f^{0.284} d^{0.588} & 14<d \leq 400\end{cases}
$$

For COST 235, which distinguishes between in-leaf (IL) and out-of-leaf (OL) situations, excess attenuation is defined as:

$$
L_{v e g \operatorname{COST}}[d B]= \begin{cases}15.6 f^{-0.009} d^{0.26} & \text { in-leaf } \\ 26.6 f^{-0.2} d^{0.5} & \text { out-of-leaf }\end{cases}
$$

To perform our comparison, based on the above we compute the corresponding expected received power $P^{r x}$ (at given distance and for a given frequency) according to Eq. (5). For the COST 235 model we consider both the in-leaf and out-of-leaf settings, given the mixed nature (i.e., coniferous/deciduous) of our forest plots.

Figure 7 compares the per-distance average of the real measurements collected in-field against the estimated received power according to Weissberger and COST 235 models, as a function of the link distance. We show only the plots for the low-power setting, both due to space constraints and because

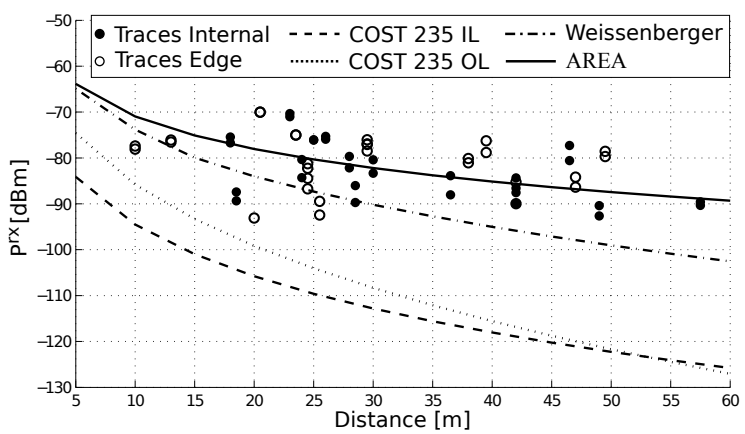

Fig. 7. Received signal power according to real RSSI traces and various prediction models (low power).

the plots for the high-power setting show similar results. COST 235 estimations, both in-leaf and out-of-leaf, do not fit well our data. Although the Weissberger model behaves slightly better, it still significantly underestimates the received power for most of the links. As a consequence, its application for network planning and deployment in this forest would likely lead to overprovisioning.

The reason behind the discrepancy between these models and the real traces is that the former are not sufficiently representative of our specific forest environment. This is not surprising, as these models are intended as generic, "one-sizefits-all" solutions, which account for vegetation by making the assumption that it is homogeneous, and as such they fail to capture the intrinsic variability displayed by the complex forest environment. In addition, these models were built for communication links that traverse the canopy (as most of the empirical models developed in the past), which is not the scenario we are considering. Finally, they have been derived empirically by curve fitting and, even though the measurement sets on which they are based is large, they clearly cannot cover all possible forest configurations at the same time.

LiDAR-based estimates: AREA. We now show that our LiDAR-based automatic approach for estimating the parameters of the model described in Section III provides more accurate predictions. Table I shows the vegetation index and its constituents for both our deployments, as estimated by the processing chain described in Section III-B. Based on these values, we compute the path loss coefficients as a function of $V D$ as per Eq. (2) and, by applying Eq. (5) and Eq. (1), we define the curve of the expected received signal power as a function of distance.

The results are shown in Figure 7. We can visually assess that the trend of our experimental data is well captured by such curve. We quantitatively evaluate the performance by comparing our in-field data and the estimated received power for the reference distances of our links. In particular, we consider the average received power computed on our low power and high power traces, in the internal and edge sites, separately. For instance, the average estimation error for the internal site at

TABLE I. VEGETATION INDEX AND ITS CONSTITUENTS IN OUR DEPLOYMENT SITES

\begin{tabular}{|c|c|c|c|}
\hline site & $D[\mathrm{~cm}]$ & $T D\left[\right.$ trees $\left./ \mathrm{m}^{2}\right]$ & $V D$ \\
\hline internal & 24.2 & 0.0355 & 0.8598 \\
edge & 25.2 & 0.0372 & 0.9366 \\
\hline
\end{tabular}




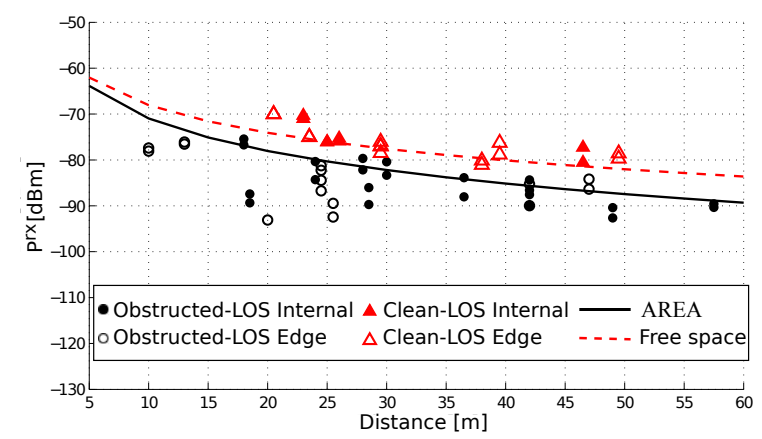

Fig. 8. Analysis of clean-LOS and obstructed-LOS links using AREA and the free space path loss model (low power).

low power is $4.06[\mathrm{dBm}]$ with a standard deviation of 3.31 , minimum 0.25 and maximum 12.1. A complete account of the estimation errors for both sites and power settings is shown in Table II.

LiDAR-based estimates: LINK. Despite the promising result, there are still significant discrepancies between the real data and the AREA prediction model. Next, we show that the LINK model we introduced in Section III-C, thanks to its ability to select the most appropriate path loss model, leads to further and significant improvement in estimation accuracy.

Figure 8 shows the real power received, as derived from our RSSI traces, for both clean-LOS (triangles) and obstructedLOS (dots) links, together with the estimation curves given by AREA and the free space path loss model. From the chart, it is evident that the latter can better predict the behavior of clean-LOS links for which, across both deployment sites, our diversified estimation strategy reduces the average error from $6.22 \mathrm{dBm}$ to $1.86 \mathrm{dBm}$ at low power, and from $14.21 \mathrm{dBm}$ to $2.71 \mathrm{dBm}$ at high power. Table II offers the complete error statistics (average, standard deviation, minimum and maximum) of this "dual-model" LINK prediction technique vs. AREA, across different deployment sites, power settings, and line-of-sight situations (clean vs. obstructed). By automatically identifying clean-LOS and obstructed-LOS links and applying our diversified strategy accordingly, we significantly and systematically reduce the estimation error for all the locations and power settings we considered in our experiments.

Figure 9 offers a different, graphical view of the comparison by plotting the real received power against the one predicted by AREA and LINK. The diagonal on the plot depicts the perfect estimate with zero error; the closer a point is to this line, the more accurate the corresponding prediction is. Moreover, the charts also show the $\pm 6 \mathrm{dBm}$ error band; we chose this value as this is the accuracy of the RSSI readings of the CC2420 radio chip our WSN nodes are equipped with. Figure 9a compares the performance of AREA and LINK w.r.t. clean-LOS and obstructed-LOS links, represented by black and white dots, respectively. It is interesting to note that AREA consistently understimates the received power for cleanLOS points. In LINK, these points are effectively "shifted" closer to the diagonal, as the effect of the attenuating $V D$ coefficient is removed from the path loss model. All of the clean-LOS points are within the $\pm 6 \mathrm{dBm}$ error band. On the other hand, AREA appears to partly overestimate the effect of vegetation. Moreover, the white points in LINK are in general less spread and much closer to the diagonal. Figure $9 \mathrm{~b}$ compares the performance of AREA and LINK w.r.t. the deployment site, i.e., internal vs. edge. In this case, LINK provides in general better predictions in both cases.

The rightmost part of Table II shows the fraction of predictions falling within the reference $\pm 6 \mathrm{dBm}$ error band for both AREA and LINK, for all the combinations of deployment sites, power settings, and link types. The quantitative data confirm that LINK systematically improves over AREA; apart from the dramatic improvement for clean-LOS links, obstructed-LOS ones have a significant improvement in the case of high power. We also show data for the narrower error band of $\pm 1 \mathrm{dBm}$, to assess how close the two models approximate the perfect estimate. Once again, LINK gets systematically closer; the best result is for clean-LOS, low-power, internal plot, where $50 \%$ of the links are within $\pm 1 \mathrm{dBm}$ of the real value with LINK, and $0 \%$ with AREA.

Although LINK represents an improvement in estimation performance, there are still discrepancies that the current model cannot account for. We argue that these are mainly due to the effect of obstructing trunks which are very close to the node, e.g., the trunks on which nodes are latched onto. This and

TABLE II. EXPERIMENTAL RESULTS

\begin{tabular}{|c|c|c|c|c|c|c|c|c|c|c|c|c|c|}
\hline & & \multicolumn{2}{|c|}{ avg error } & \multicolumn{2}{|c|}{ std dev } & \multicolumn{2}{|c|}{$\min$} & \multicolumn{2}{|c|}{$\max$} & \multicolumn{2}{|c|}{$\%$ in $\pm 6 \mathbf{d B m}$} & \multicolumn{2}{|c|}{$\%$ in $\pm 1 \mathrm{dBm}$} \\
\hline & site & AREA & LINK & AREA & LINK & AREA & LINK & AREA & LINK & AREA & LINK & AREA & LINK \\
\hline \multirow{9}{*}{ 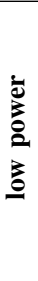 } & internal & 4.06 & 2.52 & 3.31 & 1.99 & 0.25 & 0.03 & 12.10 & 7.20 & 76.64 & 96.67 & 20.00 & 33.33 \\
\hline & clean-LOS & 6.48 & 1.97 & 2.18 & 2.08 & 4.21 & 0.03 & 9.40 & 4.94 & 50.00 & 100.00 & 0.00 & 50.00 \\
\hline & obstructed-LOS & 3.18 & 2.72 & 3.25 & 1.97 & 0.25 & 0.14 & 12.1 & 7.20 & 86.36 & 95.54 & 27.27 & 27.27 \\
\hline & edge & 5.57 & 3.11 & 3.32 & 3.02 & 0.39 & 0.40 & 15.04 & 12.50 & 56.67 & 86.67 & 6.67 & 26.67 \\
\hline & clean-LOS & 6.07 & 1.80 & 1.91 & 1.48 & 3.55 & 0.39 & 8.77 & 4.27 & 57.14 & 100.00 & 0.00 & 35.71 \\
\hline & obstructed-LOS & 5.11 & 4.33 & 4.27 & 3.58 & 0.39 & 0.39 & 15.04 & 12.50 & 56.25 & 75.00 & 12.54 & 18.75 \\
\hline & all & 4.81 & 2.81 & 3.38 & 2.55 & 0.25 & 0.03 & 15.04 & 12.50 & 66.67 & 90.00 & 13.33 & 30.00 \\
\hline & clean-LOS & 6.22 & 1.86 & 1.97 & 1.68 & 3.55 & 0.03 & 9.40 & 4.94 & 54.55 & 100.00 & 0.00 & 40.91 \\
\hline & obstructed-LOS & 3.29 & 3.37 & 3.76 & 2.81 & 0.25 & 0.14 & 15.04 & 12.50 & 73.68 & 86.84 & 21.05 & 23.68 \\
\hline \multirow{9}{*}{ 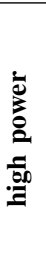 } & internal & 8.20 & 2.73 & 4.72 & 1.69 & 0.77 & 0.26 & 17.58 & 5.49 & 36.67 & 100.00 & 6.67 & 13.33 \\
\hline & clean-LOS & 14.49 & 2.94 & 2.08 & 2.06 & 12.33 & 0.83 & 17.58 & 5.49 & 0.00 & 100.00 & 0.00 & 12.50 \\
\hline & obstructed-LOS & 5.91 & 2.65 & 2.97 & 1.58 & 0.77 & 0.26 & 10.19 & 5.39 & 50.00 & 100.00 & 9.09 & 13.64 \\
\hline & edge & 10.18 & 4.15 & 4.89 & 4.97 & 0.73 & 0.43 & 17.11 & 14.12 & 30.00 & 83.33 & 3.33 & 23.33 \\
\hline & clean-LOS & 14.06 & 2.57 & 1.94 & 1.66 & 11.00 & 0.43 & 17.11 & 5.23 & 0.00 & 100.00 & 0.00 & 28.57 \\
\hline & obstructed-LOS & 6.79 & 5.53 & 4.08 & 6.40 & 0.73 & 0.42 & 14.60 & 14.12 & 56.25 & 68.75 & 6.25 & 18.75 \\
\hline & all & 9.19 & 3.44 & 4.87 & 3.75 & 0.73 & 0.26 & 17.58 & 14.12 & 33.33 & 91.67 & 5.00 & 18.33 \\
\hline & clean-LOS & 14.21 & 2.71 & 1.96 & 1.77 & 11.00 & 0.43 & 17.58 & 5.49 & 0.00 & 100.00 & 0.00 & 22.73 \\
\hline & obstructed-LOS & 6.28 & 3.86 & 3.46 & 4.48 & 0.73 & 0.26 & 14.60 & 14.12 & 52.63 & 86.84 & 7.90 & 15.79 \\
\hline
\end{tabular}




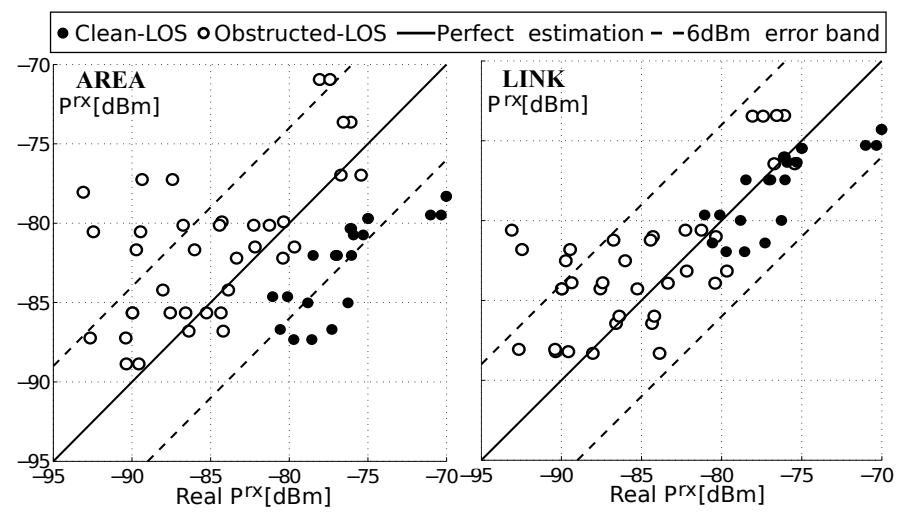

(a) Line of sight: Clean vs. obstructed.

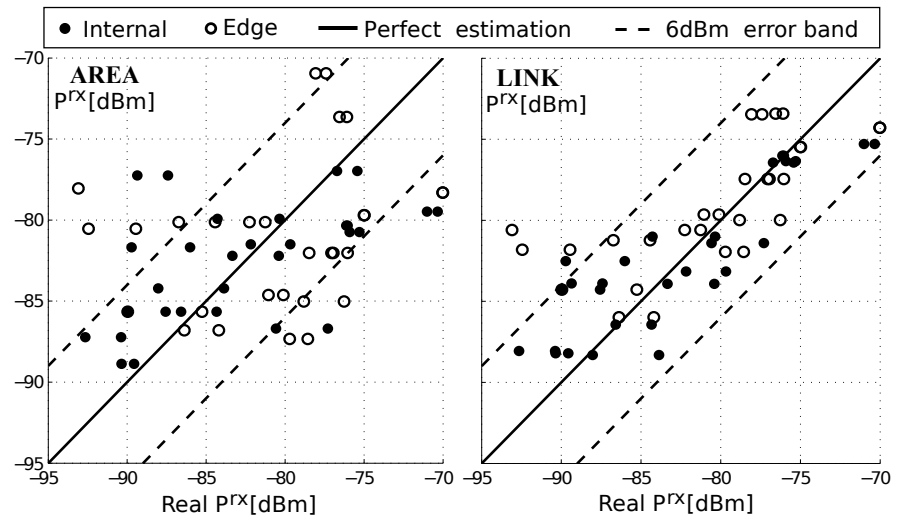

(b) Deployment site: Internal vs. edge.

Fig. 9. Prediction accuracy of AREA and LINK w.r.t. a $\pm 6 \mathrm{dBm}$ error band.

other open issues, together with possible ways to exploit RS data to deal with are addressed in Section VI.

\section{RELATED WORK}

The potential of low-power wireless communications to enable unobtrusive and dense monitoring led to a number of real-world deployments in natural scenarios [1], [2], [5]. However, the connectivity assessment before deploying the network proved to be extremely difficult in harsh environments, with both pre-deployment network planning and data interpretation practices lacking of proper support. Although the node placement problem has been addressed by the WSN community [27]-[29], the proposed approaches are often based on non-realistic assumptions (e.g., isotropic communication range), rarely satisfied in real outdoor settings. Several studies report about the experimental evidence of the environment effect on WSN links [7], [8], [30], [31], including the impact of trees on communication performance when forests are considered. This experimental evidence, together with the lack of methods for a priori connectivity assessment suitable in forest environments, motivate our investigation.

Over the last decades, several radio propagation models accounting for the presence of vegetation have been presented. The theoretical approach at the base of mechanistics models [19] involves the solution of Maxwell's equations with boundary conditions for each source of scattering along the propagation path. This approach is complex and often not applicable in practice. Therefore, several approximate and simpler empirical models, including the Weissberger [21] and COST 235 [20] models described in Section IV-D, have been developed. These models, however, are developed for scenarios in which communication links are distant from the ground and traverse the canopy. Therefore, they are not appropriate for the aforementioned common WSN applications, which rely on links that are closer to the ground and traverse the forest at the level of its trunks, as we showed in Section IV-D.

Another conventional approach is based on the log-normal path loss model [24], described in Section III-A. The critical aspect in its application is the estimation of its parameters, whose values are strictly dependent on the specific environment being considered. These parameters are usually determined empirically by regression analysis of in-field measurements. As a consequence, results are site-specific and suitable only for environments very similar to those where measurements were performed [22]. This approach has been applied for instance in [23], which specifically focused on the ISM radio bands used by WSNs, and considers propagation paths relatively near to the ground, mainly affected by trunks. The authors explicitly consider situations where trees are obstructing the line of sight between transmitter and receiver nodes, deriving distinct models for different obstruction configurations. Nevertheless, these models still lack generality, being based on regressions from location-specific measurements.

To the best of our knowledge only two works [29], [32] mention explicitly the use of remote sensing in support to WSN deployments. In [29], LiDAR is mentioned as a source of information to characterize forests and trees. However, the whereabouts of information extraction are entirely neglected; LiDAR is simply one of the possible inputs to the 3D gridbased algorithm for deploying relay nodes, which is the focus of the paper. A simplistic radio model is considered for simulations, where nodes have a fixed and isotropic transmission range that is assumed to depend on the average tree height over the area of interest.

The analysis presented in [32], instead, is closely related to our work since it focuses on RS-based techniques for path loss prediction. The authors investigate the relationship between the path loss exponent $n$ and vegetation indexes derived from Landsat 8 satellite multispectral images. These indexes (e.g., the Normalized Difference Vegetation Index, NDVI) are "greenness indicators" denoting the amount of live green vegetation. The "green" part of plants absorbs the solar radiation in the visible (red) spectral bands and reflects it in the near-infrared band; NDVI is computed as the normalized difference between the two. The spatial granularity of the analysis is inherently determined by the geometrical resolution of the images used, which represents the side of the ground area covered by each image pixel. This resolution is $30 \mathrm{~m}$ for Landsat 8-a far cry from the $50 \mathrm{~cm}$ used in our work. The work also partly uses a dataset estimated from even lowerresolution $(250 \mathrm{~m})$ MODIS images. The correlation between NDVI and path loss exponent is obtained by regression from RSSI measurements collected in a WSN deployment in an aspen boreal forest. However, this correlation appears to hold only when trees are in-leaf. Arguably, during the out-of-leaf period the amount of green leaves of the canopy is no longer the main factor affecting the communication, especially in a site characterized by deciduous trees and dense understory. In 
these conditions other factors predominate, which cannot be captured by the proposed greenness indicators.

\section{CONCLUSIONS AND FUTURE WORK}

The goal of accurately predicting the behavior of lowpower wireless communication is still a rather elusive one, especially in forests, where the irregularity of vegetation exacerbates complexity. In this paper, we presented a LiDAR-based approach that is automatic, i.e., it does not require the in-field campaigns commonly adopted to perform radio (or vegetation) surveys, and fine-grained, i.e., it enables received signal power estimates on a per-link basis. We validated our approach on real RSSI traces from two small-scale WSN deployments in a forest, and shown that it achieves unprecedented accuracy in estimating the received signal power.

Despite the encouraging results, however, further work is required to validate the approach over a broader range of scenarios (e.g., forests with different foliage or a denser understory) and environmental conditions (e.g., rain vs. dry or cold vs. warm weather). These additional experiments are already in our research agenda, and will enable us to ascertain to what extent our approach can be generalized, and how it is affected by other, complementary variables.

Other avenues for future research include the exploration of other forest attributes-e.g., the distance between a node and a tree trunk, to assess their actual impact on connectivity and consequently on our estimates - and the exploration of the cost vs. accuracy tradeoff between high-density and lowdensity LiDAR as well as other remote sensing technologies, e.g., optical systems. Finally, a practical use of our technique would be its integration in a network planning tool where, starting from an initial seed placement of nodes, the optimal placement is automatically derived.

\section{ACKNOWLEDGMENTS}

The authors would like to thank the "Dipartimento Risorse Forestali e Montane" of the Autonomous Province of Trento for providing the ortophoto and LiDAR data used in this paper in the framework of the FORLIDAR project.

\section{REFERENCES}

[1] J. Beutel et al., "PermaDAQ: A scientific instrument for precision sensing and data recovery in environmental extremes," in Proc. of IPSN, 2009

[2] A. Mainwaring et al., "Wireless sensor networks for habitat monitoring," in Proc. Workshop on Wireless Sensor Networks and Applications, 2002.

[3] M. Hefeeda and M. Bagheri, "Wireless sensor networks for early detection of forest fires," in Proc. of MASS, 2007.

[4] S. Prange et al., "New radiocollars for the detection of proximity among individuals," Wildlife Society Bulletin, vol. 34, 2006.

[5] G. P. Picco et al., "Geo-referenced Proximity Detection of Wildlife with WildScope: Design and Characterization," in Proc. of IPSN, 2015.

[6] J. K. Hart and K. Martinez, "Environmental sensor networks: A revolution in the Earth system science?" Earth-Science Reviews, vol. 78, no. 3, pp. 177-191, 2006.

[7] R. Marfievici et al., "How environmental factors impact outdoor wireless sensor networks: A case study," in Proc. of MASS, 2013.

[8] Y. Liu et al., "Does wireless sensor network scale? A measurement study on Greenorbs," IEEE Trans. Parallel Distrib. Syst., vol. 24, no. 10 , 2013.
[9] K. Srinivasan, P. Dutta, A. Tavakoli, and P. Levis, "An empirical study of low-power wireless," ACM Trans. on Sensor Networks, vol. 6, no. 2, 2010.

[10] J. A. Azevedo and F. E. Santos, "An empirical propagation model for forest environments at tree trunk level," IEEE Trans. Antennas Propag., vol. 59, no. 6, 2011.

[11] R. A. Schowengerdt, Remote sensing: models and methods for image processing. Academic press, 2006.

[12] J. Hyyppa et al., "A segmentation-based method to retrieve stem volume estimates from 3-D tree height models produced by laser scanners," IEEE Trans. Geosci. Remote Sens., vol. 39, no. 5, pp. 969-975, 2001.

[13] E. Næsset, "Predicting forest stand characteristics with airborne scanning laser using a practical two-stage procedure and field data," Remote Sens. Environ., vol. 80, no. 1, pp. 88-99, 2002.

[14] M. Heurich, "Automatic recognition and measurement of single trees based on data from airborne laser scanning over the richly structured natural forests of the bavarian forest national park," Forest Ecology and Management, vol. 255, no. 7, pp. 2416-2433, 2008.

[15] A. Kato et al., "Capturing tree crown formation through implicit surface reconstruction using airborne lidar data," Remote Sens. Environ., vol. 113, no. 6, pp. 1148-1162, 2009.

[16] C. D. Elvidge and Z. Chen, "Comparison of broad-band and narrowband red and near-infrared vegetation indices," Remote Sens. Environ., vol. 54, no. 1, pp. 38-48, 1995.

[17] M. Dalponte et al., "The role of spectral resolution and classifier complexity in the analysis of hyperspectral images of forest areas," Remote Sens. Environ., vol. 113, no. 11, pp. 2345-2355, 2009.

[18] C. Paris and L. Bruzzone, "A three-dimensional model-based approach to the estimation of the tree top height by fusing low-density lidar data and very high resolution optical images," IEEE Trans. Geosci. Remote Sens., vol. 53, no. 1, pp. 467-480, 2015.

[19] N. C. Rogers et al., "A generic model of 1-60 GHz radio propagation through vegetation-final report," Radio Agency, UK, 2002.

[20] COST 235, "Radio propagation effects on next-generation fixed-service terrestrial telecommunication systems." 1996, Luxemburg. Final Report.

[21] M. A. Weissberger, "An initial critical summary of models for predicting the attenuation of radio waves by trees," DTIC Document, Tech. Rep., 1982.

[22] T. Tamir, "On radio-wave propagation in forest environments," IEEE Trans. Antennas Propag., vol. 15, no. 6, pp. 806-817, 1967.

[23] J. A. Gay-Fernandez et al., "Propagation analysis and deployment of a wireless sensor network in a forest," Prog. Electromagn. Res., vol. 106, pp. 121-145, 2010.

[24] T. S. Rappaport, Wireless communications: Principles and Practice. Prentice Hall PTR New Jersey, 1996, vol. 2.

[25] P. Axelsson, "DEM generation from laser scanner data using adaptive TIN models," Int. Arch. Photogram. Rem. Sens., vol. 33, no. B4/1, pp. 111-118, 2000.

[26] T. Istomin et al., "Trident: In-field connectivity assessment for wireless sensor networks," Proc. of ExtremeCom, 2014.

[27] M. Younis and K. Akkaya, "Strategies and techniques for node placement in wireless sensor networks: A survey," Ad Hoc Networks, vol. 6, no. 4, 2008.

[28] A. Krause, C. Guestrin, A. Gupta, and J. Kleinberg, "Near-optimal sensor placements: Maximizing information while minimizing communication cost," in Proc. of IPSN, 2006.

[29] F. M. Al-Turjman et al., "Connectivity optimization for wireless sensor networks applied to forest monitoring," in Proc. of ICC, 2009.

[30] H. Wennerström et al., "A long-term study of correlations between meteorological conditions and 802.15.4 link performance," in Proc. of SECON, 2013

[31] M. Ceriotti et al., "Motes in the jungle: Lessons learned from a short-term WSN deployment in the Ecuador cloud forest," in Proc. of RealWSN, 2010.

[32] S. Jiang et al., "Predicting RF path loss using satellite measurements of vegetation indices," in Proc. of SenseApp, 2014. 